\title{
A Suggestion of Fuzzy Estimation Technique for Uncertainty Estimation of Linear Time Invariant System Based on Kalman Filter
}

\author{
Jong Hwa Kim ${ }^{\dagger} \cdot$ Yun $\mathrm{Su} \mathrm{Ha}{ }^{1} \cdot$ Jae Kwon $\mathrm{Lim}^{2} \cdot$ Soo Kyung Seo ${ }^{3}$ \\ (Received October 30, 2012; Revised November 13, 2012; Accepted November 19, 2012)
}

\begin{abstract}
In order to control a LTI(Linear Time Invariant) system subjected to system noise and measurement noise, first of all, it is necessary to estimate the state of system with reliability. Kalman filtering technique has been widely used to estimate the state of the stochastic LTI system with stationary noise characteristics because of its estimation ability versus algorithm simplicity. However, it often fails to estimate the state of the LTI system of which system parameter uncertainty exists partly and/or input uncertainty exists. In this paper, a new estimation technique based on Kalman filter is suggested for stochastic LTI system under parameter uncertainty and/or input uncertainty. A fuzzy estimation algorithm against uncertainties is introduced so as to compensate the state estimate filtered by Kalman filter. In order to verify the state estimation performance of the suggested technique, several simulations are accomplished.
\end{abstract}

Key words : Kalman filter, Stochastic LTI system, Uncertainty, Fuzzy estimation, Innovation process

\section{Introduction}

In order to control a LTI system subjected to system noise and measurement noise, first of all, it is necessary to estimate the state of system with reliability. Kalman filtering technique has been widely used to estimate the state of the stochastic LTI system with stationary noise characteristics [1-2]. Since Kalman gain and filter covariance are computed in off-line and they are converged to small values in steady state, the computation time is relatively short so that a digital implementation can be possible, while the estimation performance is very good.

By the way, Kalman filter often fails to estimate the state of the LTI system whose parameters are partly unknown, because Kalman filter is the model-based estimation theory and it can not completerly compensate the part of innovation process caused by the parameter errors. Then, the control system using the estimated state, by separation principle [3], can not naturally present a good control performance. Kalman filter also fails to estimate the state when unknown inputs are exerted. The reason is that Kalman filter with small Kalman gain in steady state can not sufficiently compensate the influence of unknown input although the innovation process has a large value.

In this paper, a new estimation technique is suggested, which can estimate the state of the system with reliability under uncertainties due to system parameter errors and/or unknown inputs. In order to estimate the uncertainties, a fuzzy

\footnotetext{
† Corresponding Author(Korea Maritime University, Division of IT Engineering E-mail: kimjh@hhu.ac.kr, Tel : 051-410-4343)

1 Korea Maritime University, Division of IT Engineering, E-mail: hys@hhu.ac.kr, Tel : 051-410-4347

2 Korea Maritime University, Graduate School, E-mail: jackson76@dreamwiz.com, Tel : 051-410-4894

3 Korea Maritime University, Graduate School, E-mail: soogeng426@gmail.com, Tel : 051-410-4894
} 
estimation algorithm is introduced[4]. The totally estimated uncertainty is fed back as a new input type in Kalman filter algorithm in order to compensate the estimated uncertainty. Several simulations are accomplished to explain all developing procedures and to verify the effectiveness of the suggested estimation algorithm.

\section{Parameter uncertain system and Kalman filter}

\subsection{Parameter uncertain system}

A linear LTI nominal discrete-time stochastic system is expressed by the state equation and the measurement equation given as Equation (1).

$$
\begin{aligned}
& \bar{x}_{k}=A \bar{x}_{k-1}+B u_{k-1}+D w_{k-1} \\
& \bar{z}_{k}=C \bar{x}_{k}+v_{k}
\end{aligned}
$$

where the characteristics of system noise $w_{k-1}$ and measurement noise $v_{k}$ are stationary white Gaussian as following.

$$
\begin{aligned}
& E\left[w_{k-1}\right]=0, E\left[v_{k}\right]=0, E\left[w_{k-1} v_{k}^{T}\right]=0, \\
& E\left[w_{k-1} w_{k-1}^{T}\right]=Q_{k-1}, E\left[v_{k} v_{k}^{T}\right]=R_{k}
\end{aligned}
$$

A real discrete-time system with parameter uncertainty is described as Equation (3).

$$
\begin{aligned}
& x_{k}=(A+\Delta) x_{k-1}+B u_{k-1}+D w_{k-1} \\
& z_{k}=C x_{k}+v_{k}
\end{aligned}
$$

The relationship between the nominal system and the real system is described by the state equation and measurement equation given as Equation (4).

$$
\begin{aligned}
& \bar{x}_{k}=A\left(\bar{x}_{k-1}+\tilde{x}_{k-1}\right)+B u_{k}+D w_{k-1} \\
& \tilde{x}_{k}=\Delta\left(\bar{x}_{k-1}+\tilde{x}_{k-1}\right) \\
& x_{k}=\bar{x}_{k}+\tilde{x}_{k} \\
& z_{k}=C x_{k}+v_{k}
\end{aligned}
$$

The relationship between the real system output and the nominal system output is expressed as Equation (5).

$$
\begin{aligned}
z_{k}= & C(A+\Delta)\left(\bar{x}_{k-1}+\tilde{x}_{k-1}\right)+C B u_{k-1} \\
& +C D w_{k-1}+v_{k} \\
= & C A \bar{x}_{k-1}+C B u_{k-1}+C D w_{k-1} \\
& +C \triangle \bar{x}_{k-1}+C(A+\Delta) \tilde{x}_{k-1} \\
= & \bar{z}_{k}+\tilde{z}_{k}
\end{aligned}
$$

Therefore, the real system output always contains the output error $\tilde{z}_{k}$ due to parameter uncertainty.

\subsection{Kalman filter for nominal system}

Kalman filter algorithm is the model-based estimation algorithm for LTI systems whose stochastic behaviors are stationary. Therefore, it is only implemented for the given mathematically modeled nominal system. The recursive algorithm is described based on Equation (1).

$$
\begin{aligned}
& \hat{\bar{x}}_{k}(-)=A \hat{\bar{x}}_{k-1}(+)+B u_{k-1} \\
& P_{k}(-)=A P_{k-1}(+) A^{T}+D Q_{k-1} D^{T} \\
& K_{k}=P_{k}(-) C^{T}\left[C P_{k}(-) C^{T}+R_{k}\right]^{-1} \\
& \hat{\bar{x}}_{k}(+)=\hat{\bar{x}}_{k}(-)+K_{k}\left[\bar{z}_{k}-C \hat{\bar{x}}_{k}(-)\right] \\
& P_{k}(+)=\left[I-K_{k} C\right] P_{k}(-) \\
& E\left[\bar{x}_{0}\right]=\hat{\bar{x}}_{0}, E\left[\left(\bar{x}_{0}-\hat{\bar{x}}_{0}\right)\left(\bar{x}_{0}-\hat{\bar{x}}_{0}\right)^{T}\right]=P_{0}
\end{aligned}
$$

where $\hat{\bar{x}}_{k}(-)$ means predicted state estimate, $\hat{\bar{x}}_{k}(+)$ filtered state estimate, $P_{k}(-)$ a priori covariance, $P_{k}(+)$ a posteriori covariance, and $K_{k}$ Kalman gain. $\hat{\bar{x}}_{0}$ and $P_{0}$ are initial conditions of estimated state and covariance at $t=t_{0}$. The filtered estimate $\hat{\bar{x}}_{k}(+)$ is the sum of the predicted estimate $\hat{\bar{x}}_{k}(-)$ and the compensated part multiplying Kalman gain $K_{k}$ by innovation process $\left[\bar{z}_{k}-C \hat{\bar{x}}_{k}(-)\right]$.

\subsection{Kalman filter performance test for parameter uncertain system}

Consider the following a LTI stochastic discrete -time system. 


$$
\begin{aligned}
x_{k}= & {\left[\begin{array}{rr}
0.999 & 0.009 \\
-0.247 & 0.979
\end{array}\right] x_{k-1}+\left[\begin{array}{l}
0.001 \\
0.247
\end{array}\right] u_{k-1}+\left[\begin{array}{l}
0 \\
1
\end{array}\right] w_{k-1} } \\
z_{k}= & {\left[\begin{array}{ll}
1 & 0
\end{array}\right] x_{k}+v_{k} } \\
& w_{k-1} \sim N\left(0,0.05^{2}\right), v_{k} \sim N\left(0,0.05^{2}\right)
\end{aligned}
$$

If parameter uncertainties do not exist, then Kalman filter estimates the system state normally as shown in Figure 1.
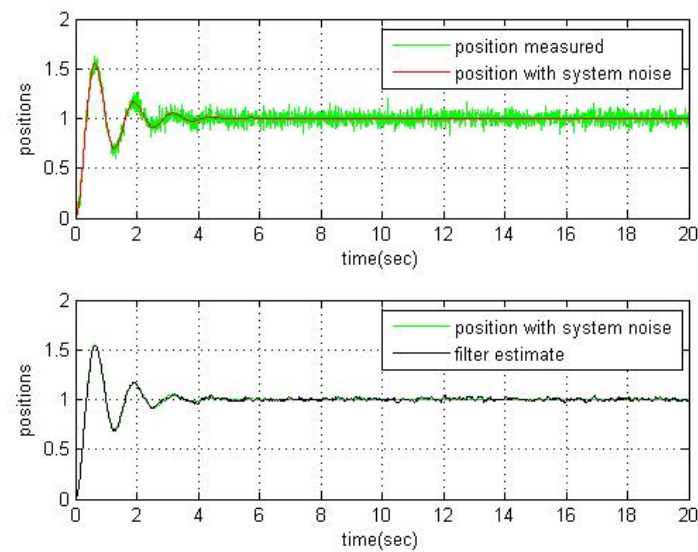

Figure 1: Nominal system output and filtered output by Kalman filter

Now consider the following a LTI stochastic discrete-time system with parameter uncertainties.

$x_{k}=\left[\begin{array}{rr}0.899 & 0.009 \\ -0.247 & 0.949\end{array}\right] x_{k-1}+\left[\begin{array}{l}0.001 \\ 0.247\end{array}\right] u_{k-1}+\left[\begin{array}{l}0 \\ 1\end{array}\right] w_{k-1}$

$A=\left[\begin{array}{rr}0.999 & 0.009 \\ -0.247 & 0.979\end{array}\right], \triangle=\left[\begin{array}{rr}-0.100 & 0.000 \\ 0.000 & 0.030\end{array}\right]$

$z_{k}=\left[\begin{array}{ll}1 & 0\end{array}\right] x_{k}+v_{k}$

$$
w_{k-1} \sim N\left(0,0.05^{2}\right), v_{k} \sim N\left(0,0.05^{2}\right)
$$

The nominal system exhibits an under-damped response in Figure 1, while the parameter uncertain exhibits an exponential growing response in Figure 2. In addition, the converged steady state values are different each other. This is often occurred when the mathematical models of ships or underwater vehicles are approximated to the linear time invariant systems, where the real models of them are composed of highly nonlinear terms expressed by hydrodynamic derivatives partly unknown $[5,6]$.
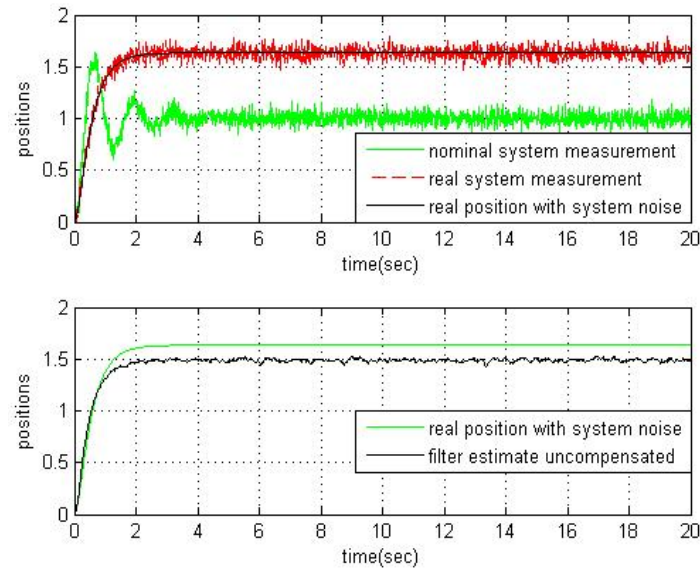

Figure 2: Parameter uncertain system output and filtered output by Kalman filter

From Figure 2, it is known that Kalman filter fails to estimate the state of the real system with parameter uncertainties. The reason is that $K_{k}$, in steady state, converges to so small value that the compensation part of Kalman filter cannot compensate the estimation error due to parameter uncertainties, in spite that the real innovation $\left[z_{k}-C \hat{\bar{x}}_{k}(-)\right]$ is a large value by substituting $\bar{z}_{k}$ by the real output $z_{k}$ in the Kalman filter algorithm.

Therefore, a new estimation algorithm to estimate the state of the parameter uncertain system is required. It is a very important problem in view of the state feedback control aspect.

\section{System subjected to unknown input and Kalman filter}

\subsection{System subjected to unknown input}

A linear LTI discrete-time stochastic system with 
unknown input is expressed by the state equation and the measurement equation given as Equation (9).

$$
\begin{gathered}
\bar{x}_{k}=A \bar{x}_{k-1}+B\left(u_{k-1}+U_{k-1}\right)+D w_{k-1} \\
\bar{z}_{k}=C \bar{x}_{k}+v_{k}
\end{gathered}
$$

where $U_{k-1}$ is an unknown input to the system at $t=(k-1) T$ and the noise characteristics are assumed to be identical to Equation (2).

The relationship between the nominal system state described by Equation (1) and the real system state described by Equation (9) is expressed as Equation (10).

$$
\begin{aligned}
x_{k} & =A x_{k-1}+B\left(u_{k-1}+U_{k-1}\right)+D w_{k-1} \\
& =A x_{k-1}+B u_{k-1}+D w_{k-1}+B U_{k-1} \\
& =\bar{x}_{k}+B U_{k-1}
\end{aligned}
$$

And the relationship between the nominal system output and the real system output can be expressed as Equation (11).

$$
\begin{aligned}
z_{k} & =C \bar{x}_{k}+C B U_{k-1}+v_{k} \\
& =C \bar{x}_{k}+v_{k}+C B U_{k-1} \\
& =\bar{z}_{k}+\tilde{z}_{k}
\end{aligned}
$$

Therefore, the real system output always contains the output error $\tilde{z}_{k}$ due to the unknown input. There are so many illustrated systems under input uncertainties. The navigation ships and underwater vehicles disturbed by wind and current for tracking the desired routes are representative illustrations and drill ships or rigs to maintain their position also good examples[5]. It is very important, in these illustrations, to estimate the magnitude of the total unknown disturbance for route tracking control as well as dynamic position control.

\subsection{Kalman filter performance test for partially unknown input system}

Simulations were accomplished to discuss the tracking ability of Kalman filter algorithm for the system with unknown input. The nominal system is the same as Equation (7). The information of inputs exerted to the system is as follows.

$$
\begin{aligned}
& u_{k-1}=1, \text { input during } t \geq 0, \\
& U_{k-1}=-1, \text { additional input during } 7 \leq t \leq 14, \\
& U_{k-1}=-0.7, \text { additional input during } t \geq 14,
\end{aligned}
$$

where the unit of time is second.

Figure 3 represents the real system output and the estimated output by Kalman filter under the assumption that the input $U_{k-1}$ is known.
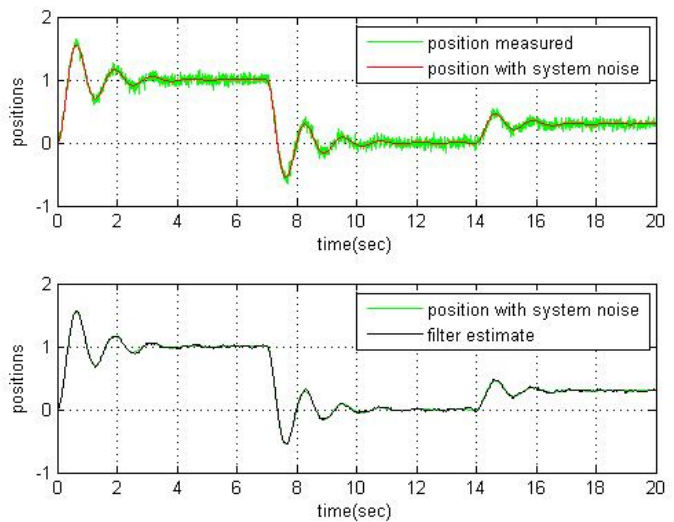

Figure 3: Filtered output by Kalman filter when input is completely known

As shown in Figure 3, Kalman filter estimates the real output with a good estimation performance substituting $u_{k-1}$ by $u_{k-1}+U_{k-1}$ in Equation (6).

Figure 4 represents the real system output and the estimated output by Kalman filter under the assumption that the input $U_{k-1}$ is unknown. In this case, Kalman filter fails to estimate the real system output and thus the steady state estimation error is revealed.

In this aspect, a new estimation algorithm to estimate the state of the system with unknown input, is required. 

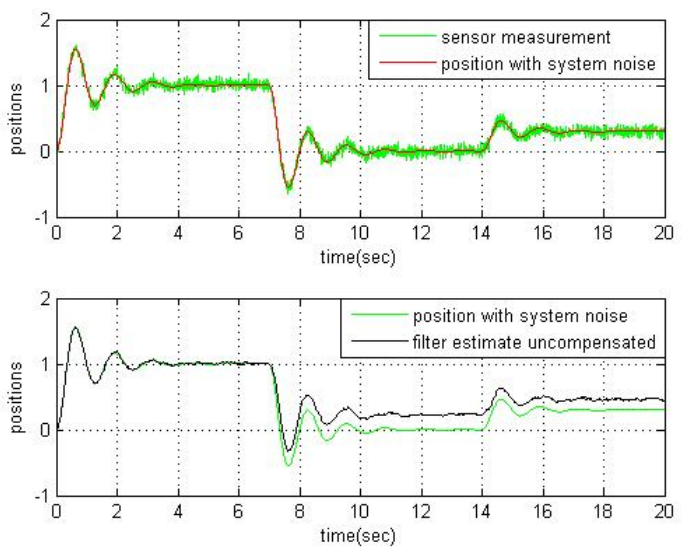

Figure 4: Filtered output by Kalman filter when additional input $U_{k-1}$ is unknown

\section{Suggestion of a new estimation algorithm for uncertainties}

4.1 Suggestion of a fuzzy estimation algorithm to estimate Uncertainties

In this section, a fuzzy estimation algorithm for estimating the amount of uncertainties which causes the estimation error of Kalman filter is suggested.

Figure 5 is the structure of the fuzzy estimation algorithm [6-7]. The basic information for the fuzzy estimation algorithm is the innovation process $r_{k}$ which is the real system output $z_{k}$ subtracted by the predicted output $\hat{\bar{z}}_{k}$.

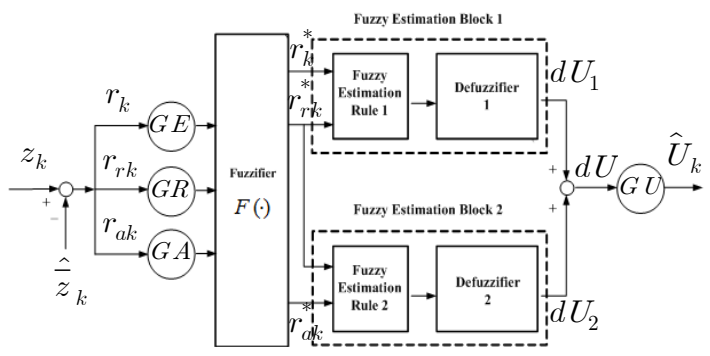

Figure 5: Structure of the suggested fuzzy estimation algorithm

The notations used in Figure 5 are as follows.

$$
\begin{aligned}
& r_{k}=z_{k}-\hat{\bar{z}}_{k}, r_{k}^{*}=G E \times r_{k} \\
& r_{r k}=r_{k} / T, r_{r k}^{*}=G R \times r_{r k} \\
& r_{a k}=\left[r_{k}-r_{k-1}\right] / T, r_{a k}^{*}=G A \times r_{a k} \\
& G E=L / r_{k}, G R=L / r_{r k}, G A=L / r_{a k} \\
& G U=4 / G R, d U=d U_{1}+d U_{2} \\
& \hat{U}_{k}=G U \times d U
\end{aligned}
$$

Here $r_{k}$ is innovation, $r_{r k}$ is $r_{k}$ divided by $T, r_{a k}$ is rate of $r_{k}$, and $T$ is sampling time. $G E, G R$, and $G A$ are input scalers for normalizing fuzzy input $r_{k}, r_{r k}$, and $r_{a k}$. $G U$ is output scaler of the fuzzy output $d U . \hat{U}_{k}$ is the estimated uncertainty.

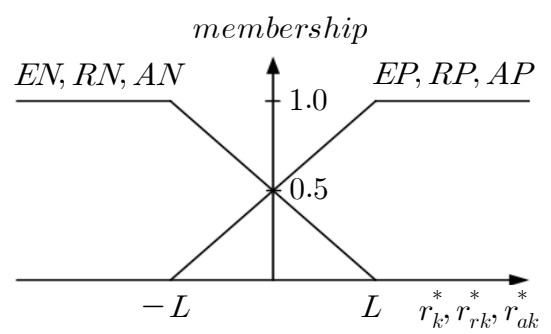

Figure 6: Input fuzzifications for the fuzzy estimation algorithm

$L$ is the design parameter for normalizing inputs.

Figure 6 represents the input fuzzifications of the suggested fuzzy estimation algorithm and Figure 7 represents the output fuzzifications of the suggested fuzzy estimation algorithm.

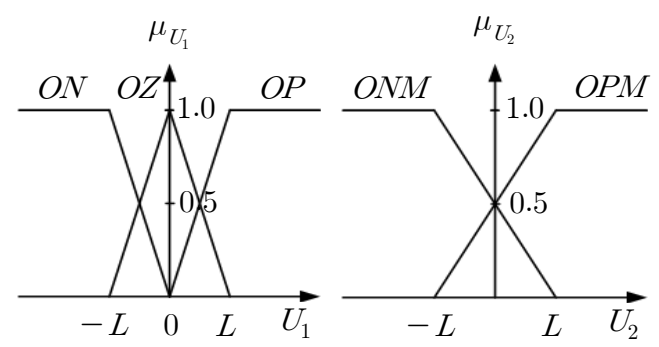

Figure 7: Output fuzzifications for the fuzzy estimation algorithm 
Fuzzy estimation rules of the fuzzy estimation block 1 for uncertainty estimation are as follows.

$$
\begin{aligned}
& (R 1)_{1}: I F r_{k}^{*}=E P \text { and } r_{r k}^{*}=R P, U_{1}=O P \\
& (R 2)_{1}: I F r_{k}^{*}=E P \text { and } r_{r k}^{*}=R N, U_{1}=O Z \\
& (R 3)_{1}: I F r_{k}^{*}=E N \text { and } r_{r k}^{*}=R P, U_{1}=O Z \\
& (R 4)_{1}: I F r_{k}^{*}=E N \text { and } r_{r k}^{*}=R N, U_{1}=O N
\end{aligned}
$$

Fuzzy estimation rules of the fuzzy estimation block 1 for uncertainty estimation are as follows.

$$
\begin{aligned}
& (R 1)_{2}: I F r_{r k}^{*}=R P \text { and } r_{a k}^{*}=A P, U_{2}=O P M \\
& (R 2)_{2}: I F r_{r k}^{*}=R P \text { and } r_{a k}^{*}=A N, U_{2}=O N M \\
& (R 3)_{2}: I F r_{r k}^{*}=R N \text { and } r_{a k}^{*}=A P, U_{2}=O P M \\
& (R 4)_{2}: I F r_{r k}^{*}=R N \text { and } r_{a k}^{*}=A N, U_{2}=O N M
\end{aligned}
$$

$U_{1}$ and $U_{2}$ stand for the fuzzy outputs of fuzzy rules in the fuzzy estimation block 1 and block 2, respectively.

If the center of area method as defuzzification algorithms is applied to each estimation block to compute $d U_{i}(i=1,2)$ and the results are arranged, the estimated uncertainty $\hat{U}_{k}$ is computed by Equation (13).

$$
\begin{aligned}
\hat{U}_{k} & =K_{i} r_{k}+K_{p} r_{r k}+K_{d} r_{a k} \\
K_{i} & =0.5 \times G U \times G E \\
K_{p} & =0.5 \times G U \times G R \\
K_{d} & =0.25 \times G U \times G A
\end{aligned}
$$

The procedure to obtain the result Equation (13) based on Figure 5 is addressed precisely in $[6,7]$.

\subsection{Test for uncertainty presence}

As discussed in section 2 and 3, Kalman filter estimates the state of the LTI system with reliability in case uncertainties do not exist. In this case, the fuzzy estimation algorithm is no longer needed and it is desirable to use Kalman filter only. When uncertainties exist in the system, Kalman filter fails to estimate the real state and the value of innovation process increases.

In this paper, a method based on innovation process is used as a test method of uncertainty presence. Figure $\mathbf{8}$ shows the test method on uncertainty presence.

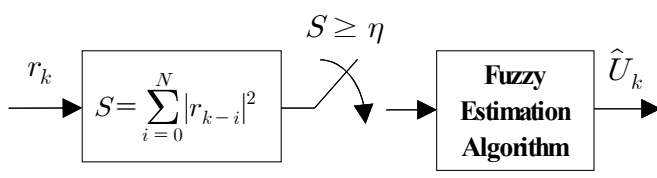

Figure 8: Test for uncertainty presence

$S$ is the sum of square of $r_{k-i}$ for $i=0,1, \cdots, N$. $N$ is a finite window length and $\eta$ is a threshold that are decided by a fuzzy estimation algorithm designer, under considering the performance, the sensitivity, and the stability in generating $\hat{U}_{k}$ simultaneously.

\subsection{A new estimation algorithm based on Kalman filter}

The total amount of uncertainty caused by partially unknown parameters and unknown inputs can be estimated by the suggested fuzzy estimation algorithm. By using this estimated amount, a new estimation algorithm based on Kalman filter can be composed, in order to estimate the state of the stochastic LTI system subjected to uncertainties.

$$
\begin{aligned}
& \hat{x}_{k}(-)=A \hat{x}_{k-1}(+)+B\left(u_{k-1}+\hat{U}_{k-1}\right) \\
& P_{k}(-)=A P_{k-1}(+) A^{T}+D Q_{k-1} D^{T} \\
& K_{k}=P_{k}(-) C^{T}\left[C P_{k}(-) C^{T}+R_{k}\right]^{-1} \\
& \hat{x}_{k}(+)=\hat{x}_{k}(-)+K_{k}\left[z_{k}-C \hat{x}_{k}(-)\right] \\
& P_{k}(+)=\left[I-K_{k} C\right] P_{k}(-) \\
& \hat{U}_{k}=K_{i} r_{k}+K_{p} r_{r k}+K_{d} r_{a k}
\end{aligned}
$$

The nominal state $\hat{\bar{x}}_{k}$ of Kalman filter based on 
the mathematical model is substituted by the state $\hat{x}_{k}$ compensated by the estimated uncertainty. The nominal measurement output $\hat{\bar{z}}$ is also substituted by the output $\hat{z}_{k}$ compensated by the estimated uncertainty.

\section{Simulations to verify the estimation performance}

Several simulations were executed to verify the estimation performance of the suggested estimation algorithm under uncertainties.

It was shown that Kalman filter failed to estimate the state of the system with parameter uncertainties in Figure 2. Figure 9 shows the simulation result when the suggested estimation algorithm is applied to the uncertain parameter system given by Equation (8). The first figure is the estimation result of the standard Kalman filter and the second is the result of the suggested estimation algorithm. As shown in figure, the suggested method estimates the real state of the system with parameter uncertainties.
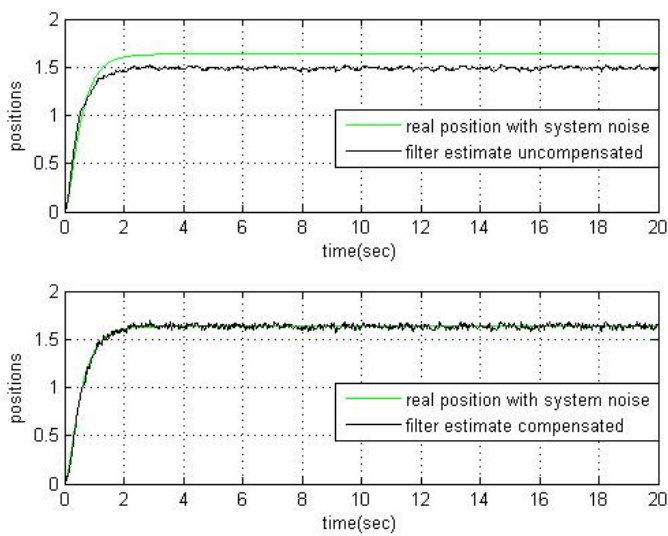

Figure 9: The estimation result of the suggested algorithm for parameter uncertain system

In case that the unknown inputs are exerted on the stochastic LTI system, Kalman filter failed to estimate the real state of the system as shown in
Figure 4. Figure 10 shows the simulation result when the suggested estimation algorithm is applied to the same system with unknown input.
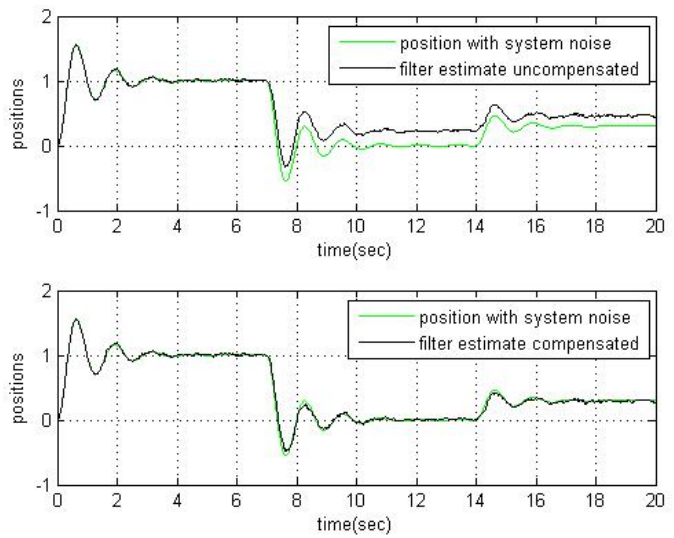

Figure 10: The estimation result of the suggested algorithm for unknown input system

The first of Figure $\mathbf{1 0}$ is the estimation result of the standard Kalman filter and the second is the result of the suggested estimation algorithm. As shown in figure, the suggested method estimates the real state of the system with the unknown input.

\section{Conclusions}

In this paper, a new estimation algorithm based on Kalman filter was suggested. Through several simulations, it was verified that the suggested method can estimate the state of the system under uncertainties such as parameter uncertainties and/or unknown inputs. A fuzzy estimation algorithm plays an important role to estimate the amount of uncertainty. The total estimated uncertainty was fed back as if a new input were exerted to Kalman filter algorithm.

\section{References}

[1] A. E. Gelb et al., Applied Optimal Estimation, MIT Press, Cambridge, 1974.

[2] M. S. Grewal and A. P. Andrew, Kalman Filtering Theory and Practice, Prentice-Hall, 
New Jersey, 1993.

[3] A. P. Sage and C. C. White, Optimum Systems Control, Prentice-Hall, Seoul, 1977.

[4] J. Kim, J. Lim, S. Seo, and Y. Ha, "Kalman filter based estimation algorithm for uncertainty estimation of LTI system," Proceedings of the 36th KOSME Fall Conference, p. 157, 2012 (in Korean).

[5] T. I. Fossen, Marine Control Systems : Guidance, Navigation, and Control of Ships, Rigs, and Underwater Vehicles, Marine Cybernetics, Trondheim Norway, 2002.

[6] I. Kim, B. Lee, and J. Kim, "A study on the performance improvement of the nonlinear fuzzy PID controller," Journal of Korean Society of Marine Engineers, vol. 27, no. 7, pp. 852-861, 2003 (in Korean).

[7] I. H. Kim, "A Study on the Design Method of a Nonlinear Fuzzy PID Controller with Variable Parameters," Ph.D, Thesis, Department of Control and Instrumentation Engineering, Korea Maritime University, 2004 (in Korean). 